HU-EP-01/17

hep-th/0104156

\title{
Curved BPS domain wall solutions in five-dimensional gauged supergravity
}

\author{
Gabriel Lopes Cardoso, Gianguido Dall'Agata and Dieter Lüst \\ Institut für Physik, Humboldt University \\ Invalidenstraße 110, 10115 Berlin, Germany \\ email:gcardoso, dallagat, luest@physik.hu-berlin.de
}

\begin{abstract}
We analyze the possibility of constructing supersymmetric curved domain wall solutions in five-dimensional $\mathcal{N}=2$ gauged supergravity, which are supported by non-constant scalar fields belonging either to vector multiplets only or to vector and hypermultiplets. We show that the BPS equations for the warp factor and for the vector scalars are modified by the presence of a four-dimensional cosmological constant on the domain wall, in agreement with earlier results by DeWolfe, Freedman, Gubser and Karch. We also show that the cosmological constant on the domain wall is anti-de Sitter like and that it constitutes an independent quantity, not related to any of the objects appearing in the context of very special geometry.
\end{abstract}




\section{Introduction}

The idea [1] that our four-dimensional universe is just a domain wall embedded in five-dimensional spacetime has attracted a lot of attention recently [2]-[6]. So far, the study of BPS domain wall solutions in five-dimensional gauged supergravity has been restricted to the case of flat four-dimensional domain walls [7]-[15]. However, on general grounds, it is quite natural to ask whether there also exist curved BPS domain walls in five-dimensional gauged supergravity, i.e. four-dimensional curved domain walls with a non-vanishing cosmological constant. Such curved domain walls seem to play an important role in the context of locally localized gravity [16] 18 in spaces with infinite volume.

In four dimensions, curved BPS domain wall solutions were recently constructed [19] in the context of $\mathcal{N}=2$ gauged supergravity coupled to vector multiplets. There it was shown that an anti-de Sitter cosmological constant on the domain wall is related to both the imaginary part of the superpotential and to a non-vanishing $U(1)$-connection. Such a non-vanishing connection is also known to give rise to rotating four-dimensional BPS black hole solutions [20, 21]. Since this $U(1)$-connection is expressed in terms of a set of harmonic functions that also determine the behaviour of the scalar fields belonging to the vector multiplets, the anti-de Sitter cosmological constant on the domain wall does not constitute an independent quantity.

On the other hand, in $\mathcal{N}=2$ gauged supergravity in five dimensions, there is no such $U(1)$-connection due to the reality property of very special geometry and hence, a mechanism similar to the one discussed above is not available. Nevertheless, as we will show in this paper, it may still be possible to construct curved domain wall solutions which are supported by non-constant scalar fields belonging either to vector multiplets only or to vector and hypermultiplets (we do not discuss the case of curved domain wall solutions with non-constant hyper scalar fields only). To achieve this, one has to give up the requirement that the potential of the theory be written solely in terms of a real superpotential $W$. The resulting BPS equations for the warp factor and for the vector scalars are then modified by the presence of a four-dimensional cosmological constant on the domain wall. These modified flow equations are in agreement with the earlier findings of [22], which were established in the context of non-supersymmetric gravitational theories in five dimensions. We will also show that the cosmological constant on the domain wall is then necessarily anti-de Sitter like and that it constitutes

an independent quantity, not related to any of the objects appearing in the context of very special geometry. This is again in analogy with the angular momentum carried by five-dimensional rotating BPS black hole solutions in the theory of $\mathcal{N}=2$ ungauged supergravity coupled to vector multiplets, where the angular momentum enters as an independent quantity in the expression for the macroscopic Bekenstein-Hawking entropy [23].

In the case that all of the scalars are taken to be constant, the resulting solution 
describes an $\mathcal{N}=2$ five-dimensional anti-de Sitter vacuum with an (anti-)de Sitter foliation along the radial coordinate as in [22].

\section{Setup}

The five-dimensional $\mathcal{N}=2$ gauged supergravity theory that we will consider in the following is the one constructed in [24], describing the general coupling of $n_{V}$ abelian vector multiplets and of $n_{H}$ hypermultiplets to supergravity. The scalar fields $\phi^{x}(x=$ $\left.1, \ldots, n_{V}\right)$ of the vector multiplets parametrize a very special manifold, whose sections $h^{I}(\phi)$ satisfy the constraint 25

$$
C_{I J K} h^{I} h^{J} h^{K}=1
$$

with real $C_{I J K}\left(I=0, \ldots, n_{V}\right)$. The dual fields $h_{I}(\phi)$ are defined by

$$
h_{I}=C_{I J K} h^{J} h^{K} .
$$

The hypermultiplet scalars $q^{X}$, on the other hand, parametrize a quaternionic Kähler geometry determined by $4 n_{H}$-beins $f_{X}^{i A}\left(q^{X}\right)$, with the $S U(2)$ index $i=1,2$ and the $S p\left(2 n_{H}\right)$ index $A=1, \ldots, 2 n_{H}$, raised and lowered by the symplectic metrics $C_{A B}$ and $\varepsilon_{i j}$. We refer to [24 for more details.

We will be interested in the construction of BPS solutions with spacetime metrics given by

$$
d s^{2}=\mathrm{e}^{2 U(r)} \hat{g}_{m n} d x^{m} d x^{n}+\mathrm{e}^{-2 p U(r)} d r^{2}
$$

with $\hat{g}_{m n}=\hat{g}_{m n}\left(x^{m}\right)$. Here we denote spacetime indices by $x^{\mu}=\left(x^{m}, r\right)$ and the corresponding tangent space indices by $a=(0,1,2,3,5)$. The constant $p$ is introduced for later convenience. We assume Lorentz invariance in the four-dimensional subspace $a=(0,1,2,3)$. The metric $\hat{g}_{m n}$ is thus a four-dimensional constant curvature metric,

$$
\hat{R}_{m n}=-\frac{12}{l^{2}} \hat{g}_{m n}
$$

with the four-dimensional cosmological constant proportional to $l^{-2}$. The case of (imaginary) real $l$ corresponds to a four-dimensional (anti-) de Sitter spacetime. We take the solutions to be uncharged, that is we set the gauge fields to zero. We allow for a nontrivial dependence of the scalar fields on the coordinate $r$, and we write $\phi^{\prime}=\partial \phi / \partial r$, $q^{\prime}=\partial q / \partial r$ as well as $U^{\prime}=\partial U / \partial r$.

In the absence of gauge fields, the supersymmetry transformation laws for the gravitini $\psi_{\mu i}$, for the gaugini $\lambda_{i}^{x}$ and for the hyperini $\zeta^{A} \operatorname{read}$ [24, 15]

$$
\begin{aligned}
\delta \psi_{\mu i} & =D_{\mu}(\omega) \epsilon_{i}+\frac{i}{\sqrt{6}} g \gamma_{\mu} P_{i j} \epsilon^{j} \\
\delta \lambda_{i}^{x} & =-\frac{i}{2}\left(\not \partial \phi^{x}\right) \epsilon_{i}+g P_{i j}^{x} \epsilon^{j}, \\
\delta \zeta^{A} & =-\frac{i}{2} f_{i X}^{A}\left(\not \partial q^{X}\right) \epsilon^{i}+g \mathcal{N}_{i}^{A} \epsilon^{i},
\end{aligned}
$$


where (as for all triplets $P_{i j}=i P^{s}\left(\sigma^{s}\right)_{i j}$ )

$$
\begin{array}{rlrl}
P^{s} & \equiv h^{I}(\phi) P_{I}^{s}(q), & P_{x}^{s} \equiv-\sqrt{\frac{3}{2}} \partial_{x} P^{s}=h_{x}^{I} P_{I}^{s}, \\
\mathcal{N}_{i}^{A} & \equiv \frac{\sqrt{6}}{4} f_{i X}^{A} K^{X}, & K^{X} & \equiv h^{I}(\phi) K_{I}^{X}(q) .
\end{array}
$$

The scalar potential of such a theory is given by the squares of the shifts of the supersymmetry transformations

$$
\mathcal{V}=-4 P^{s} P^{s}+2 P_{x}^{s} P_{y}^{s} g^{x y}+2 \mathcal{N}_{i A} \mathcal{N}^{i A}
$$

and depends only on the triplet of prepotentials $P^{s}$ and their derivatives. By decomposing the triplet $P^{s}$ into its norm and phases,

$$
P^{s}=\sqrt{\frac{3}{2}} W Q^{s}, \quad Q^{s} Q^{s}=1,
$$

one obtains

$$
W=h^{I}(\phi) P_{I} \quad, \quad P_{I} \equiv \sqrt{\frac{2}{3}} P_{I}^{s}(q) Q^{s}
$$

as well as [15]

$$
\mathcal{V}=-6 W^{2}+\frac{9}{2} g^{\Lambda \Sigma} \partial_{\Lambda} W \partial_{\Sigma} W+\frac{9}{2} W^{2}\left(\partial_{x} Q^{s}\right)\left(\partial^{x} Q^{s}\right)
$$

Here $g_{\Lambda \Sigma}$ denotes the metric of the complete scalar manifold, which is positive definite, involving the scalars of both the vector and the hypermultiplets. The derivatives acting on the $S U(2)$ phases $Q^{s}$ are just computed with respect to the scalars of the vector multiplets. As noted in [15], when $\partial^{x} Q^{s}=0$, i.e. when the phases only depend on the quaternions (or when they are constant), then the potential goes into a form for which gravitational stability is guaranteed [26].

We can now compute the integrability conditions coming from the vanishing of the gravitini variation (5). As already stated above, we only allow for a non-trivial dependence of the scalar fields on the coordinate $r$. This results in the following integrability conditions (for $p=0$ ):

$$
\begin{aligned}
R_{m n} & =\left[4 g^{2} W^{2}-\frac{1}{3} \phi^{x \prime} \phi^{y \prime} g_{x y}(\phi)-\frac{1}{6} q^{X \prime} q^{Y \prime} g_{X Y}(q)-\frac{1}{4} K^{X} K^{Y} g_{X Y}(q)\right] \mathrm{e}^{2 U} \hat{g}_{m n},(13) \\
R_{r r} & =4 g^{2} W^{2}-\frac{4}{3} \phi^{x \prime} \phi^{y \prime} g_{x y}(\phi)-\frac{2}{3} q^{X \prime} q^{Y \prime} g_{X Y}(q)-K^{X} K^{Y} g_{X Y}(q)
\end{aligned}
$$

where we also made use of the vanishing of the transformations (6) and (77).

For a metric of the form (3) subject to (4) this yields

$$
\begin{aligned}
3 U^{\prime \prime}+\frac{12}{l^{2}} \mathrm{e}^{-2 U} & =-\phi^{x \prime} \phi^{y \prime} g_{x y}-\frac{1}{2} q^{X \prime} q^{Y \prime} g_{X Y}-\frac{9}{2} g^{2} g^{X Y} \partial_{X} W \partial_{Y} W, \\
\left(U^{\prime}\right)^{2}-\frac{4}{l^{2}} \mathrm{e}^{-2 U} & =g^{2} W^{2},
\end{aligned}
$$


where we used that $g_{X Y} K^{X} K^{Y}=6 g^{X Y} \partial_{X} W \partial_{Y} W$. For supersymmetric configurations, these equations are equivalent to the Einstein equations. It follows that the BPS equations we will obtain below must be compatible with (15) and (16). From (16) we obtain that

$$
U^{\prime}= \pm \gamma(r) g W
$$

where

$$
\gamma(r)=\sqrt{1+\frac{4 \mathrm{e}^{-2 U}}{l^{2} g^{2} W^{2}}} .
$$

The first order differential equation (17) for the warp factor $\mathrm{e}^{U}$ was also derived in 22 in the context of non-supersymmetric five-dimensional gravity theories.

\section{$3 \quad$ Flat BPS domain walls}

The case of flat BPS domain walls without hypermultiplets has already been discussed extensively in the literature [10]-[13]. Flat BPS domain walls with hypermultiplets have been discussed in [12, 14, 15]. Here we briefly review some of the features of these solutions, and we present an extension of these results to the case of non-trivial hypermultiplets.

Inserting the spacetime line element (3) into the gravitini variation yields

$$
\begin{aligned}
\delta \psi_{m i} & =\hat{\mathcal{D}}_{m} \epsilon_{i}+\frac{1}{2} \mathrm{e}^{p U} \partial_{r} U \gamma_{m} \gamma_{5} \epsilon_{i}+\frac{i}{2} g W Q_{i j} \gamma_{m} \epsilon^{j} \\
\delta \psi_{r i} & =\partial_{r} \epsilon_{i}-q^{X \prime} \omega_{X}{ }^{j} \epsilon_{j}+\frac{i}{2} g W \mathrm{e}^{-p U} Q_{i j} \gamma_{5} \epsilon^{j}
\end{aligned}
$$

where $\hat{\mathcal{D}}_{m}$ denotes the covariant derivative with respect to the metric $\hat{g}_{m n}$.

First consider the variation $\delta \psi_{m i}=0$. Since the four-dimensional domain wall is flat $(\gamma=1)$, we set

$$
\hat{\mathcal{D}}_{m} \epsilon_{i}=0 \text {. }
$$

We then obtain from $\delta \psi_{m i}=0$ that

$$
\frac{1}{2} \mathrm{e}^{p U} \partial_{r} U \gamma_{5} \epsilon_{i}=-\frac{i}{2} g W Q_{i j} \epsilon^{j} .
$$

The consistency of (22) implies that

$$
\mathrm{e}^{2 p U}\left(\partial_{r} U\right)^{2}=g^{2} W^{2}
$$

and hence

$$
\mathrm{e}^{p U} \partial_{r} U= \pm g W
$$


which is in accordance with (17). Inserting (24) into (22) yields the projector condition

$$
i \gamma_{5} \epsilon_{i}= \pm Q_{i j} \epsilon^{j}
$$

Since this is the only condition we impose on the supersymmetry parameters, we conclude that the resulting flat domain wall solutions preserve $1 / 2$ of $\mathcal{N}=2$ supersymmetry.

Next, let us consider the vanishing of the variation of the other fermion fields. Using the projector equation (25), it follows from (6) and (17) that the scalar fields $\phi^{\Lambda}=\left\{\phi^{x}, q^{X}\right\}$ have to satisfy 15

$$
\phi^{\Lambda^{\prime}}=\mp 3 g \mathrm{e}^{-p U} g^{\Lambda \Sigma} \partial_{\Sigma} W
$$

We note that (26) and (24) imply (15).

Consistency of the above equations also gives the following constraint on the phases $Q^{s}[15$

$$
\partial_{x} Q^{s}=0
$$

Thus, it follows from (11) that $W=h^{I}(\phi) P_{I}(q)$. Using the chain rule $\partial_{r} h_{I}=\partial_{x} h_{I} \phi^{x \prime}$ as well as (24), one then obtains from (26) that

$$
\mathrm{e}^{p U} \partial_{r} h_{I}(\phi)+2 \mathrm{e}^{p U}\left(\partial_{r} U\right) h_{I}(\phi)= \pm 2 g P_{I}(q)
$$

We note that (28) implies (24). This follows simply by contracting (28) with $h^{I}$ and using that $h^{I} h_{I}=1$ as well as its consequence $h^{I} \partial_{r} h_{I}=0$.

Next, we introduce the rescaled fields

$$
Y^{I}=\mathrm{e}^{U} h^{I} \quad, \quad Y_{I}=\mathrm{e}^{2 U} h_{I},
$$

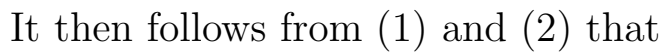

$$
\mathrm{e}^{3 U}=C_{I J K} Y^{I} Y^{J} Y^{K} \quad, \quad Y_{I}=C_{I J K} Y^{J} Y^{K} .
$$

Setting

$$
p=2
$$

for convenience [12], we then obtain from (28) that

$$
\partial_{r} Y_{I}= \pm 2 g P_{I}(q)
$$

which is solved by

$$
Y_{I}=c_{I} \pm 2 g \int^{r} d r^{\prime} P_{I}(q)
$$


where the real $c_{I}$ denote integration constants. A solution to this equation in closed form can only be given if one determines the behaviour of the quaternions $q^{X}$. It does not appear to be possible to determine the behaviour of $P_{I}(q)$ in a way that is independent from the vector multiplet scalars.

When only vector multiplets are present, and in the case of Abelian gaugings, the superpotential $W$ reduces to $W=h^{I} \alpha_{I}$, where $\alpha_{I}$ are the Fayet-Iliopoulos terms, which are constant. Then it follows that [12]

$$
Y_{I}=c_{I} \pm 2 g \alpha_{I} r
$$

These equations are called attractor equations.

\section{Curved BPS domain walls}

Now we take $\hat{g}_{m n}$ to be a constant curvature metric satisfying (4). Then (21) gets modified, and a natural ansatz describing this modification is given by

$$
\hat{\mathcal{D}}_{m} \epsilon_{i}=f(r) \hat{e}_{m}{ }^{a} \gamma_{a} \gamma_{5} \epsilon_{i}+\frac{i}{2} h(r) Q_{i j} \hat{e}_{m}{ }^{a} \gamma_{a} \epsilon^{j}
$$

where $f(r)$ and $h(r)$ denote real functions. Equation (35) gives rise to the following integrability condition:

$$
f^{2}(r)-\frac{1}{4} h^{2}(r)=\frac{1}{l^{2}}
$$

As we shall see momentarily, the simple choices $f^{2}=l^{-2}, h=0$ and $f=0, h^{2}=-4 l^{-2}$ for the case of real and imaginary $l$, respectively, do not lead to a supersymmetric solution.

We set $p=0$ in the following. Inserting (35) into the supersymmetry condition $\delta \psi_{m i}=$ 0 now yields

$$
\left(\mathrm{e}^{-U} f(r)+\frac{1}{2} \partial_{r} U\right) \gamma_{5} \epsilon_{i}=-\frac{i}{2}\left(\mathrm{e}^{-U} h(r)+g W\right) Q_{i j} \epsilon^{j}
$$

The consistency of (37) implies that

$$
\mathrm{e}^{-U} f(r)+\frac{1}{2} \partial_{r} U= \pm \frac{1}{2}\left(\mathrm{e}^{-U} h(r)+g W\right)
$$

Then, combining (38) with (36) and using (17) yields

$$
f(r)=\mp \frac{1}{2} \mathrm{e}^{U} \gamma g W \quad, \quad h(r)=-\mathrm{e}^{U} g W,
$$

with $\gamma$ given by (18). We note that when inserting (39) into (37) both sides of the latter equation vanish, so that (37) does not result in a restriction of the amount of supersymmetry preserved by the solution. 
In the case of constant scalar fields, the superpotential $W$ is constant and equation (17) can be solved explicitly. For $\gamma \neq 0$ and $|l| \neq \infty$ the solution reads

$$
\mathrm{e}^{U}=\frac{2}{\lg W} \sinh \left( \pm g W\left(r-r_{0}\right)\right)
$$

for real $l$, whereas for imaginary $l$ it is given by

$$
\mathrm{e}^{U}=\frac{2}{|l| g W} \cosh \left( \pm g W\left(r-r_{0}\right)\right)
$$

As shown in [22], this describes a four-dimensional (anti-)de Sitter foliation of the $\mathcal{N}=2$ supersymmetric $A d S_{5}$ vacuum. It can be checked that (15) is satisfied by this solution. The case $\gamma=0$, on the other hand, does not lead to a solution of (15).

Let us now turn to the case of non-constant scalar fields. First consider the case when $\partial_{x} Q^{s}=0$. Below we will show that, in order to construct non-trivial solutions to the gaugino variation equation $\delta \lambda_{i}^{x}=0$ preserving $1 / 2$ of $\mathcal{N}=2$ supersymmetry, one has to impose the projector condition (25) on the supersymmetry parameters. Inspection of (37) then shows that $f=h=0$, so that $l^{-1}=0$ and $\gamma=1$, which gives rise to a flat domain wall solution.

Thus, in order to obtain curved BPS domain wall solutions with either non-constant vector scalar fields or non-constant vector and hyper scalar fields甲, one has to allow for a dependence of the $Q^{s}$ on some of the vector multiplet scalars?. We will shortly see that this implies that the projector condition (25) has to get modified, as follows:

$$
i \gamma_{5} \epsilon_{i}=A(r) Q_{i}{ }^{j} \epsilon_{j}+B(r) M_{i}{ }^{j} \epsilon_{j}
$$

The $S U(2)$-valued matrix $Q=i Q^{s} \sigma^{s}$ contains the phases of the prepotentials $P_{i j}$ (as in (10)), which may now depend on both the vector and the hyper scalars, and $M=i M^{s} \sigma^{s}$ denotes an $S U(2)$-valued matrix satisfying $M_{i}{ }^{j} M_{j}{ }^{k}=-\delta_{i}{ }^{k}$ (i.e. $M^{s} M^{s}=1$ ). Without loss of generality, we take $Q$ and $M$ to be orthogonal in $S U(2)$ space, so that $Q^{s} M^{s}=0$. The consistency of (42) then yields that

$$
A^{2}(r)+B^{2}(r)=1
$$

Since (42) is the only condition we will impose on the supersymmetry parameters, the resulting curved domain wall solutions will preserve $1 / 2$ of $\mathcal{N}=2$ supersymmetry.

Inserting (42) into the gaugini variation equation $\delta \lambda_{i}^{x}=0$ yields (with $p=0$ )

$$
\left(A(r) Q_{i}{ }^{j} \epsilon_{j}+B(r) M_{i}{ }^{j} \epsilon_{j}\right) \phi^{x \prime}=\sqrt{6} g g^{x y} \partial_{y} P_{i}{ }^{j} \epsilon_{j}
$$

\footnotetext{
${ }^{\mathrm{a}}$ It may be possible to construct curved domain wall solutions with only non-constant hyper scalars turned on [27.

${ }^{\mathrm{b}}$ In $\mathcal{N}=2$ gauged supergravity coupled to vector multiplets only, it may be possible to achieve $\partial_{x} Q^{s} \neq 0$ by a non-abelian gauging.
} 
Then, using the decomposition of $P^{s}$ given in (10) yields

$$
\left(A(r) Q^{s}+B(r) M^{s}\right) \phi^{x \prime}=3 g g^{x y}\left(Q^{s} \partial_{y} W+W \partial_{y} Q^{s}\right) .
$$

Since $Q^{s} \partial_{x} Q^{s}=0$, the two pieces on the right hand side of this equation are orthogonal to each other, and hence it follows that

$$
A(r) \phi^{x \prime}=3 g g^{x y} \partial_{y} W
$$

as well as

$$
B(r) M^{s} \phi^{x \prime}=3 g W g^{x y} \partial_{y} Q^{s} .
$$

Inspection of (47) shows that if the $Q^{s}$ do not depend on any of the vector scalar fields, then either all the vector scalar fields are constant, or $B M^{s}=0$. In the latter case, the projector condition (42) reduces to (25), thus yielding flat domain wall solutions. Hence we take $\partial_{\tilde{x}} Q^{s} \neq 0$ in the following. We denote the subset of vector scalar fields, on which the $Q^{s}$ depend, by $\phi^{\tilde{x}}$. The remaining vector scalars will be denoted by $\phi^{\hat{x}}$. In order to be able to solve (46) and (47), we take the metric $g_{x y}$ to be factorisable as $g_{x y}=\left(g_{\hat{x} \hat{y}}, g_{\tilde{x} \tilde{y}}\right)$. Then, we find from (46) and (47) that

$$
\phi^{\hat{x}}=\text { constant }, \partial_{\hat{x}} W=0 \text {. }
$$

The scalar fields $\phi^{\tilde{x}}$, on the other hand, are non-constant.

Squaring (47) and using $M^{s} M^{s}=1$ yields

$$
B^{2}(r)=9 g^{2} W^{2} \frac{g^{\tilde{x} \tilde{y}}\left(\partial_{\tilde{x}} Q^{s}\right)\left(\partial_{\tilde{y}} Q^{s}\right)}{g_{\tilde{x} \tilde{y}} \phi^{\tilde{x}^{\prime}} \phi^{\tilde{y} \prime}} .
$$

Since the right hand side of (49) is positive definite due to the reality of the $Q^{s}$ and the positivity of the metric $g_{\tilde{x} \tilde{y}}$, we conclude that $B(r)$ is a real function.

On the other hand, we obtain from (47) that

$$
M^{s}=\frac{3 g W}{B} \frac{\phi^{\tilde{x} \prime} \partial_{\tilde{x}} Q^{s}}{g_{\tilde{x} \tilde{y}} \phi^{\tilde{x} \prime} \phi^{\tilde{y} \prime}} .
$$

Squaring this and using again that $M^{s} M^{s}=1$ yields

$$
B^{2}(r)=9 g^{2} W^{2} \frac{\left(\phi^{\tilde{x} \prime} \partial_{\tilde{x}} Q^{s}\right)\left(\phi^{\tilde{y} \prime} \partial_{\tilde{y}} Q^{s}\right)}{\left(g_{\tilde{x} \tilde{y}} \phi^{\tilde{x} \prime} \phi^{\tilde{y} \prime}\right)^{2}} .
$$

Equating (51) with (49) then yields

$$
\left(\phi^{\tilde{x} \prime} \partial_{\tilde{x}} Q^{s}\right)\left(\phi^{\tilde{y} \prime} \partial_{\tilde{y}} Q^{s}\right)=\left(g_{\tilde{x} \tilde{y}} \phi^{\tilde{x} \prime} \phi^{\tilde{y} \prime}\right)\left(g^{\tilde{z} \tilde{w}}\left(\partial_{\tilde{z}} Q^{s}\right)\left(\partial_{\tilde{w}} Q^{s}\right)\right) .
$$

We note that in the case that $Q^{s}$ only depends on one scalar field $\phi^{\tilde{x}}$, then the relation (52) is automatically satisfied. 
We now determine $A(r)$ by using the integrability condition resulting from the gravitini equation $\delta \psi_{m i}=0$. Inserting (42) into (19) yields

$$
\hat{\mathcal{D}}_{m} \epsilon_{i}=\frac{i}{2}\left(U^{\prime} A+g W\right) \gamma_{m} Q_{i}{ }^{j} \epsilon_{j}+\frac{i}{2} U^{\prime} B \gamma_{m} M_{i}{ }^{j} \epsilon_{j},
$$

whose integrability gives

$$
\left(U^{\prime} A+g W\right)^{2}+B^{2}\left(U^{\prime}\right)^{2}=-4 l^{-2} \mathrm{e}^{-2 U} .
$$

Inserting (43) as well as (17) into (54) then gives

$$
A(r)=\mp \gamma(r) \text {. }
$$

Since the left hand side of (54) is positive definite, we conclude that $l$ has to be purely imaginary. Thus we conclude that the presence of non-constant vector scalars excludes the possibility of having BPS de Sitter like domain walls.

Inserting (55) into (46) yields

$$
\phi^{\tilde{x} \prime}=\mp 3 g \gamma^{-1} g^{\tilde{x} \tilde{y}} \partial_{\tilde{y}} W
$$

Comparison of (26) with (56) shows that, in the presence of an anti-de Sitter cosmological constant on the wall, the flow equation for the vector scalar fields $\phi^{\tilde{x}}$ gets modified by a factor $\gamma^{-1}$. This is in agreement with a similar finding 222 in the context of non-supersymmetric gravity theories in five dimensions.

We note that in the case of constant hyper scalars, (56) and (17) imply (15).

Using the chain rule $\partial_{r} h_{I}=\partial_{x} h_{I} \phi^{x^{\prime}}=\partial_{\tilde{x}} h_{I} \phi^{\tilde{x}^{\prime}}$ as well as (17), one obtains from (56) that

$$
\partial_{r} h_{I}(\phi)+2 \gamma^{-2}\left(\partial_{r} U\right) h_{I}(\phi)= \pm 2 g \gamma^{-1} P_{I} .
$$

We note that (57) implies (17). This follows simply by contracting (57) with $h^{I}$ and using that $h^{I} h_{I}=1$ as well as $h^{I} \partial_{r} h_{I}=0$.

Introducing the rescaled fields

$$
Y^{I}=\mathrm{e}^{\int^{r} d r^{\prime} \gamma^{-2}\left(r^{\prime}\right)\left(\partial_{r^{\prime}} U\right)} h^{I} \quad, \quad Y_{I}=\mathrm{e}^{2 \int^{r} d r^{\prime} \gamma^{-2}\left(r^{\prime}\right)\left(\partial_{r^{\prime}} U\right)} h_{I}
$$

yields

$$
\partial_{r} Y_{I}= \pm 2 g \gamma^{-1} \mathrm{e}^{2 \int^{r} d r^{\prime} \gamma^{-2}\left(r^{\prime}\right)\left(\partial_{r^{\prime}} U\right)} P_{I}
$$

Under the coordinate change $d r \rightarrow \mathrm{e}^{-2 \int^{r} d r^{\prime} \gamma^{-2}\left(r^{\prime}\right)\left(\partial_{r^{\prime}} U\right)} d r$ this goes into

$$
\partial_{r} Y_{I}= \pm 2 g \gamma^{-1} P_{I}
$$


and hence

$$
Y_{I}=c_{I} \pm 2 g \int^{r} d r^{\prime} \gamma^{-1}\left(r^{\prime}\right) P_{I},
$$

where the real $c_{I}$ denote integration constants. An equation for the warp factor analogous to (30) can be obtained by inserting (58) into (11). An explicit solution for the warp factor may then be derived by expanding in powers of $l^{-1}$.

We also obtain from (49), (55), (56) and (43) that

$$
g^{\tilde{x} \tilde{y}}\left(\partial_{\tilde{x}} Q^{s}\right)\left(\partial_{\tilde{y}} Q^{s}\right)=g^{\tilde{x} \tilde{y}}\left(\partial_{\tilde{x}} W\right)\left(\partial_{\tilde{y}} W\right) \frac{\left(1-\gamma^{2}\right)}{\gamma^{2} W^{2}} .
$$

Inserting (62) into the expression for the potential (12) then yields

$$
\mathcal{V}=-6 W^{2}+\frac{9}{2} \gamma^{-2} g^{\tilde{x} \tilde{y}} \partial_{\tilde{x}} W \partial_{\tilde{y}} W+\frac{9}{2} g^{X Y} \partial_{X} W \partial_{Y} W,
$$

where we also used (48). The expression (63) for the potential is in agreement with the one given in [22].

Finally, let us briefly comment on the vanishing of the hyperini variation, $\delta \zeta^{A}=0$. The insertion of (42) into $\delta \zeta^{A}=0$ results in a more complicated matrix equation than (26), with the metric $g^{X Y}$ in (26) replaced by a more complicated object. We leave the analysis of the resulting matrix equation for the future.

To summarize, we have addressed the issue of the construction of curved BPS domain wall solutions in five dimensions. We have seen that in the case when such domain wall solutions are supported either by non-constant vector scalars or by both non-constant vector and hyper scalars, we have to require $\partial_{\tilde{x}} Q^{s} \neq 0$. We have also seen that only antide Sitter domain walls are allowed by this construction. The resulting first order BPS equations for the warp factor and for the vector scalars ((17) and (56), respectively) are modified by the factor $\gamma$ which depends on the four-dimensional cosmological constant on the wall. These equations agree with those obtained previously in [22] in the context of non-supersymmetric five-dimensional gravity theories. We also note the presence of the additional condition (52) on the solution, as well as those arising from the flow equations for the hyper scalars which we haven't analyzed in this paper.

In the presence of hypermultiplets, it has been shown [15] that a gauged supergravity theory can possess critical points with ultraviolet and infrared directions. Using the AdS/CFT correspondence, this leads to the possibility of the construction of domain wall solutions dual to regular renormalization group flows. It would be very interesting to see if it is possible to construct curved BPS domain wall solutions interpolating between such vacua and to understand the meaning of the domain wall cosmological constant in the dual field theory flow. As an example one could consider deforming the two-parameter solution of [15] describing the $\mathcal{N}=2$ embedding of the UV-IR solution of [9]. 


\section{Acknowledgements}

We would like to thank K. Behrndt, P. Breitenlohner, B. de Wit and D. Maison for valuable discussions. We are very much indepted to A. Karch, L. Randall and A. Strominger for pointing out an error in the previous version of this paper. This work is supported by the DFG and by the European Commission RTN Programme HPRNCT-2000-00131.

\section{References}

[1] V. A. Rubakov and M. E. Shaposhnikov, "Do We Live Inside A Domain Wall?," Phys. Lett. B125 (1983) 136-138.

[2] A. Lukas, B. A. Ovrut, K. S. Stelle and D. Waldram, "The universe as a domain wall," Phys. Rev. D59 (1999) 086001, hep-th/9803235.

[3] N. Arkani-Hamed, S. Dimopoulos and G. Dvali, "The hierarchy problem and new dimensions at a millimeter," Phys. Lett. B429 (1998) 263-272, hep-th/9803315.

[4] I. Antoniadis, N. Arkani-Hamed, S. Dimopoulos and G. Dvali, "New dimensions at a millimeter to a Fermi and superstrings at a TeV," Phys. Lett. B436 (1998) 257-263, hep-th/9804398.

[5] L. Randall and R. Sundrum, "A large mass hierarchy from a small extra dimension," Phys. Rev. Lett. 83 (1999) 3370-3373, hep-th/9905221.

[6] L. Randall and R. Sundrum, "An alternative to compactification," Phys. Rev. Lett. 83 (1999) 4690-4693, hep-th/9906064.

[7] A. Lukas, B. A. Ovrut, K. S. Stelle, and D. Waldram, "Heterotic M-theory in five dimensions," Nucl. Phys. B552 (1999) 246-290, hep-th/9806051.

[8] L. Girardello, M. Petrini, M. Porrati, and A. Zaffaroni, "Novel local CFT and exact results on perturbations of $N=4$ super Yang-Mills from AdS dynamics," JHEP 12 (1998) 022, hep-th/9810126.

[9] D. Z. Freedman, S. S. Gubser, K. Pilch and N. P. Warner, "Renormalization group flows from holography supersymmetry and a c-theorem," Adv. Theor. Math. Phys. 3 (1999) 363-417, hep-th/9904017.

[10] K. Behrndt and M. Cvetic, "Supersymmetric domain wall world from D = 5 simple gauged supergravity," Phys. Lett. B475 (2000) 253-260, hep-th/9909058.

[11] R. Kallosh and A. Linde, "Supersymmetry and the Brane World," JHEP 0002 (2000) 005, hep-th/0001071. 
[12] K. Behrndt and S. Gukov, "Domain walls and superpotentials from M theory on Calabi- Yau three-folds," Nucl. Phys. B580 (2000) 225-242, hep-th/0001082.

[13] K. Behrndt and M. Cvetic, "Anti-de sitter vacua of gauged supergravities with 8 supercharges," Phys. Rev. D61 (2000) 101901, hep-th/0001159.

[14] K. Behrndt, "A non-singular infra-red flow from $D=5$ gauged supergravity,", Phys. Lett. B487 (2000) 30-35, hep-th/0005185.

[15] A. Ceresole, G. Dall'Agata, R. Kallosh and A. Van Proeyen, "Hypermultiplets, domain walls and supersymmetric attractors," hep-th/0104056.

[16] A. Karch and L. Randall, "Locally localized gravity," Int. J. Mod. Phys. A16 (2001) 780-800, hep-th/0011156.

[17] A. Karch and L. Randall, "Localized Gravity in String Theory," hep-th/0105108.

[18] A. Karch and L. Randall, "Open and Closed String Interpretation of SUSY CFT's on Branes with Boundaries," hep-th/0105132.

[19] K. Behrndt, G. L. Cardoso and D. Lüst, "Curved BPS domain wall solutions in four-dimensional $\mathrm{N}=2$ supergravity," hep-th/0102128.

[20] K. Behrndt, D. Lüst and W. A. Sabra, "Stationary solutions of N=2 supergravity," Nucl. Phys. B510 (1998) 264-288, hep-th/9705169.

[21] G. L. Cardoso, B. de Wit, J. Käppeli, and T. Mohaupt, "Stationary BPS solutions in $\mathrm{N}=2$ supergravity with $R^{2}$ interactions," JHEP 0012 (2000) 019, hep-th/0009234.

[22] O. DeWolfe, D. Z. Freedman, S. S. Gubser and A. Karch, "Modeling the fifth dimension with scalars and gravity," Phys. Rev. D62 (2000) 046008, hep-th/9909134.

[23] A. H. Chamseddine and W. A. Sabra, "Metrics Admitting Killing Spinors In Five Dimensions," Phys. Lett. B426 (1998) 36-42, hep-th/9801161.

[24] A. Ceresole and G. Dall'Agata, "General matter coupled $\mathcal{N}=2, D=5$ gauged supergravity," Nucl. Phys. B585 (2000) 143-170, hep-th/0004111.

[25] M. Günaydin, G. Sierra and P. K. Townsend, "The geometry of N=2 Maxwell-Einstein supergravity and Jordan algebras," Nucl. Phys. B242 (1984) 244.

[26] P.K. Townsend, "Positive energy and the scalar potential in higher dimensional (super)gravity theories," Phys. Lett. B148 (1984) 55.

[27] K. Behrndt and M. Cvetic, work in progress. 